\title{
No Association Between MTHFR A1298C and MTRR A66G Polymorphisms, and MS in an Australian Cohort
}

\author{
A.L. Szvetko ${ }^{a}$, J. Fowdar ${ }^{a}$, J. Nelson ${ }^{a}$, N. Colson ${ }^{a}$, L. Tajouri, \\ P.A. Csurhes ${ }^{b}$, M.P. Pender ${ }^{b, c}$ and L.R. Griffiths ${ }^{a}$ \\ ${ }^{\mathrm{a}}$ Genomics Research Centre, School of Medical Science, Griffith University, Gold Coast, PMB 50, \\ Gold Coast Mail Centre, Queensland 9726, Australia \\ ${ }^{\mathrm{b}}$ Neuroimmunology Research Centre, School of Medicine, University of Queensland, Australia \\ ${ }^{\mathrm{c}}$ Department of Neurology, Royal Brisbane and Women's Hospital, Brisbane, Australia
}

\begin{abstract}
Multiple sclerosis (MS) is a complex neurological disease that affects the central nervous system (CNS) resulting in debilitating neuropathology. Pathogenesis is primarily defined by CNS inflammation and demyelination of nerve axons. Methionine synthase reductase (MTRR) is an enzyme that catalyzes the remethylation of homocysteine (Hcy) to methionine via cobalamin and folate dependant reactions. Cobalamin acts as an intermediate methyl carrier between methylenetetrahydrofolate reductase (MTHFR) and Hcy. MTRR plays a critical role in maintaining cobalamin in an active form and is consequently an important determinant of total plasma Hcy (pHcy) concentrations. Elevated intracellular pHcy levels have been suggested to play a role in CNS dysfunction, neurodegenerative, and cerebrovascular diseases. Our investigation entailed the genotyping of a cohort of 140 cases and matched controls for MTRR and MTHFR, by restriction length polymorphism (RFLP) techniques. Two polymorphisms: MTRR A66G and MTHFR A1298C were investigated in an Australian age and gender matched case-control study. No significant allelic frequency difference was observed between cases and controls at the $\alpha=0.05$ level (MTRR $\chi^{2}=0.005$, $P=0.95$, MTHFR $\chi^{2}=1.15, P=0.28$ ). Our preliminary findings suggest no association between the MTRR A66G and MTHFR A1298C polymorphisms and MS.
\end{abstract}

Keywords: Multiple sclerosis; Gene association; MTHFR; MTRR; Homocysteine

\section{Introduction}

Multiple sclerosis (MS) is a complex neurological disease that affects the central nervous system (CNS) resulting in debilitating neuropathology. Pathogenesis is primarily defined by CNS inflammation and demyelination of nerve axons. The precise etiology of MS remains elusive with a complex interplay between environmental factors, genetic susceptibility, and age-dependant exposure to viral infection postulated [1]. A significant genetic component has been demonstrated by studies examining familial concordance rates with risk being up to 300fold increased in monozygotic twins and 20-40-fold increased for first-degree relatives as compared to a general population prevalence of $0.1 \%$ [2].

Methionine synthase reductase (MTRR) is an enzyme that catalyzes the remethylation of homocysteine (Hcy) to methionine via a cobalamin and folate dependant reaction. Hcy is an intermediate in the folate, vitamin B12, and B6 dependent pathways of one-carbon and sulfur amino acid metabolism [3]. Cobalamin acts as an intermediate methyl carrier between methylenetetrahydrofolate reductase (MTHFR) and Hcy [4]. The cobalamin cofactor cycles between cob(I)alamin and methylcob(III)alamin. Cob(I)alamin is a strong reductant and over time becomes oxidised to produce an inactive cob(II)alamin form of methionine synthase [5]. MTRR plays a critical role in maintaining cobalamin in an active 
form and is consequently an important determinant of total plasma Hcy (pHcy) concentration. Elevated intracellular pHcy has been implicated in CNS dysfunction, neurodegenerative, and cerebrovascular disease. Dysfunction is thought to result from vasotoxic and neurotoxic effects of elevated Hcy, inhibition of the methylation of myelin basic protein (MBP) and membrane phospholipids, and disruption of biogenic amine metabolism [6].

The A1298C polymorphism in the MTHFR gene has been associated with hyperhomocysteineemia [7]. The A1298C allele is characterized by a point mutation at position 1298 of the MTHFR gene (located 1p36.3) causing the replacement of glutamine by alanine in the corresponding enzyme [8]. The A66G polymorphism in the gene MTRR (located 5p15.3) alters an isoleucine into a methionine residue. The 66G variant may constitute a functional mutation associated with a reduced reductive repair of methionine synthase due to the crucial localization of methionine synthase reductase in the enzyme [9]. We present an investigation of the possible association of the MTHFR A1298C and MTRR A66G polymorphisms with MS in a case-control study of an Australian cohort.

\section{Materials and methods}

\subsection{Study population}

Griffith University's Ethics Committee approved research involving human experimentation. Informed consent was obtained from all participants. The case-control populations consisted of 140 MS patients and 140 healthy controls, which had been matched for age, gender, and ethnicity (Caucasian). The MS population was initially obtained from the Multiple Sclerosis Clinic at the Royal Brisbane Hospital; all samples were procured from patients residing in the South East Queensland region. The populations consisted of 75\% females and 25\% males, and were subcategorized based on clinical course: Relapsing-Remitting (RR), SecondaryProgressive (SP), and Primary-Progressive (PP). Frequencies were: $\mathrm{RR}=40 \%$, SP $=36 \%$, and $\mathrm{PP}=24 \%$. The matched control (age \pm 5 years, gender, and ethnicity) population samples were obtained via the Genomics Research Centre Clinic, Southport. Genomic DNA was extracted from peripheral blood using a standard salting-out procedure.

\subsection{Genotyping}

Genomic DNA was used as a template to generate polymerase chain reaction (PCR) products for subsequent restriction enzyme digest and genotyping. Kara et al. and Feix et al. have previously described PCR primers for MTHFR and MTRR, respectively and adaptations of their genotyping methods were used for this study [10] and [3]. Primers for MTHFR were: forward 5'-CTT TGG GGA GCT GAA GGA CTA CTA C-3', reverse 5'-CAC TTT GTG ACC ATT CCG GTT TG-3'. Primers for MTRR were: forward 5'-GCA AAG GCC ATC GCA GAA GAC AT-3', reverse 5'-AAA CGG TAA AAT CCA CTG TAA CGG C-3'. Primers were obtained from Proligo Primers and Probes (Sigma-Aldrich). Assay conditions were optimised with thermal cycling conditions for MTHFR consisting of $95^{\circ} \mathrm{C}$ for $3 \mathrm{~min}$, then 40 cycles of $94^{\circ} \mathrm{C}$ for $1 \mathrm{~min}, 60^{\circ} \mathrm{C}$ for $1 \mathrm{~min}, 72^{\circ} \mathrm{C}$ for $1 \mathrm{~min}$, with a final extension of $72{ }^{\circ} \mathrm{C}$ for $7 \mathrm{~min}$, and MTRR consisting of $95^{\circ} \mathrm{C}$ for $2 \mathrm{~min}$, then 40 cycles of $95^{\circ} \mathrm{C}$ for $1 \mathrm{~min}$, $60{ }^{\circ} \mathrm{C}$ for $1 \mathrm{~min}, 72{ }^{\circ} \mathrm{C}$ for $1 \mathrm{~min}$, with a final extension of $72{ }^{\circ} \mathrm{C}$ for $2 \mathrm{~min}$. MTHFR and MTRR PCR products were resolved on a $2 \%$, and $5 \%$ high range agarose gel (Progen Biosciences) yielding $163 \mathrm{bp}$ and $118 \mathrm{bp}$ amplicons, respectively. Restriction enzyme digest of the MTHFR product was with MboII (Fermentas Life Science), and the MTRR product with $N s p$ I (New England Biolabs). MTHFR A1298A produced five fragments: 56, 31, 30, 28, and $18 \mathrm{bp}$, whilst A1298C produced six fragments: 84, 56, 31, 30, 28, and $18 \mathrm{bp}$. MTHFR digest products were resolved using an $8 \%$ resolution plus agarose gel (Progen Biosciences). The MTRR sense-primer introduced a mismatch at the 3rd nucleotide (from the 3' end) creating a restriction site for the $N s p$ I enzyme whenever the $G$ allele was present. In the 
presence of the A allele the $118 \mathrm{bp}$ product remained uncleaved, in the presence of the $\mathrm{G}$ allele two fragments were produced: 24 and $94 \mathrm{bp}$. MTRR digest products were resolved using a 5\% resolution plus agarose gel (Progen Biosciences).

\section{Statistical analysis}

Descriptive statistics were explored using the SPSS Standard Package (Version 11.0 for Mac OS X). Genotype and allele frequencies were investigated using standard Chi-square $\left(\chi^{2}\right)$ analysis using Microsoft Excel. Genotype distributions were checked for Hardy-Weinberg equilibrium.

\subsection{Exclusion criteria for statistical analysis}

Of the cohort of 140 cases/controls, 10 cases and 9 matched controls (MTHFR) and 17 cases and 16 matched controls (MTRR) were excluded due to genotyping difficulties, leaving 130 cases and 131 matched controls (MTHFR) and 123 cases and 124 matched controls (MTRR) for final analysis.

TABLE 1. Genotype and allele frequencies for MTHFR

\begin{tabular}{|l|l|l|l|l|l|l|l|}
\hline & \multicolumn{3}{|l|}{ Genotype frequencies } & N & \multicolumn{4}{l|}{ Allele frequencies } \\
\hline & AA & AC & CC & & A & C & $N$ \\
\hline Control & $63(48 \%)$ & $49(37 \%)$ & $19(15 \%)$ & 131 & $175(67 \%)$ & $87(33 \%)$ & 262 \\
\hline MS & $53(41 \%)$ & $56(43 \%)$ & $21(16 \%)$ & 130 & $162(62 \%)$ & $98(38 \%)$ & 260 \\
\hline & $\chi^{2}=1.42, P=0.49(\alpha=0.05)$ & & $\chi^{2}=1.15, P=0.28(\alpha=0.05)$ \\
\hline
\end{tabular}

TABLE 2. Genotype and allele frequencies for MTRR

\begin{tabular}{|l|l|l|l|l|l|l|l|}
\hline & \multicolumn{3}{|l|}{ Genotype frequencies } & N & \multicolumn{3}{l|}{ Allele frequencies } \\
\hline & AA & AG & GG & & A & G & $N$ \\
\hline Control & $47(38 \%)$ & $64(52 \%)$ & $13(10 \%)$ & 124 & $158(64 \%)$ & $90(36 \%)$ & 248 \\
\hline MS & $49(40 \%)$ & $58(47 \%)$ & $16(13 \%)$ & 123 & $156(63 \%)$ & $90(37 \%)$ & 246 \\
\hline & $\chi^{2}=0.64, P=0.73(\alpha=0.05)$ & & $\chi^{2}=0.005, P=0.95(\alpha=0.05)$ \\
\hline
\end{tabular}

\section{Results}

Genotypes were determined after PCR amplification and restriction enzyme cutting prior to standard agarose gel electrophoresis. Results for genotype and allele frequency are detailed in Table 1 and Table 2. Genotype frequencies for both the case and control groups, for both markers conformed to Hardy-Weinberg equilibrium expectations. The observed control frequencies for this study also conformed with independent studies [3] and [10]. There was no statistically significant difference between case and control allele frequencies for either the 
MTHFR $\left(\chi^{2}=1.15, P=0.28, \alpha=0.05\right)$; or MTRR $\left(\chi^{2}=0.005, P=0.95, \alpha=0.05\right)$; tested variants. For MTHFR, the A allele was more frequently observed than the $C$ allele with very similar count in the cases and controls ( $\mathrm{A}=0.67$ and 0.62$)$. Similarly, for the MTRR variant, the A allele was more frequent than the $\mathrm{G}$ allele $(\mathrm{A}=0.64$ and 0.63$)$ but similar in both cases and controls.

\section{Discussion}

MS remains a paradigm of complex disease with variable clinical expression and unpredictable course. Disease susceptibility is partly genetically determined, however the underlying molecular mechanisms of MS pathogenesis remain unidentified [11]. In vitro studies have demonstrated the ability of Hcy to induce neurotoxicity through over-stimulation of $N$-methyl-d-aspartate (NMDA) receptors resulting in neuronal damage due to excessive $\mathrm{Ca}^{2+}$ influx and induction of reactive oxygen species [12]. MTHFR deficiency is the most common genetic cause of hyperhomocysteinemia [13]. Homocysteine may also sensitize neurons to oxidative stress via oxidation of sulfhydryl groups subsequently resulting in generation of reactive oxygen species such as superoxide and hydrogen peroxide [14]. The A1298C mutation in MTHFR has previously been associated with hyperhomocyteinemia [7]. MTRR plays a crucial role in homocysteine metabolism and is necessary to maintain the function of methionine synthase [15]. It has previously been hypothesized that the MTRR A66G variant may constitute a functional mutation associated with a reduced reductive repair of methionine synthase due to the crucial localization of methionine synthase reductase in the enzyme [9].

We investigated the MTHFR A1298C and the MTRR A66G variants for association with MS in an Australian case-control cohort. The findings of this study suggest no association with the two tested markers and MS in our population. No statistically significant difference was observed by genotype or allele frequencies for either marker between our cases and controls. Despite these findings, it is possible that other variants in MTHFR and MTRR may be associated with MS or in fact other neurodegenerative disorders. The MTHFR variant C66T has been reported to be positively associated with schizophrenia [16]. An American study further supports the association that a folate-sensitive defect in homocysteine metabolism contributes to cases of that disease [17]. An Irish study of 601 participants has reported a significant association between elevated pHcy levels and another variant of MTRR, A66G [4]. Lindenbaum and colleagues have suggested that measurement of pHcy levels before and after treatment can be valuable for confirmation of a differential diagnosis of neuropsychiatric disorder in patients presenting with paresthesia, sensory loss, ataxia, and dementia [18]. A recent study that confirmed elevated pHcy levels in MS patients concluded that the finding is unlikely to be related to immune activation, oxidative stress, or deficiencies in vitamin B6, B12, or folate [19]. Other investigations also support the finding of elevated homocysteine levels in MS patients compared with healthy controls [20], [21] and [22]. It is hypothesized that the nervous system may be particularly sensitive to pHcy as it promotes excitotoxicity by stimulation of NMDA receptors and damages neuronal DNA hence triggering apoptosis in neuronal cells [14] and [23]. Ergo, although our findings suggest no association between MTHFR A1298C and MTRR A66G and MS, other variants associated with the homocysteine metabolism cascade may still contribute to MS susceptibility.

\section{Acknowledgements}

This research was supported by a Trish Multiple Sclerosis Research Foundation (in association with Multiple Sclerosis Australia and MS Research Australia) Postgraduate Scholarship for Attila Szvetko and a Griffith University Research Grant. The research undertaken complied with Australian legislation and ethical standards, and was approved by the Griffith University Ethics Committee. 


\section{References}

[1] L.I. Levin, K.L. Munger, M.V. Ruberstone, C.A. Peck, E.T. Lennette and D. Spiegelmen et al., Temporal relationship between elevation of Epstein-Barr virus antibody titers and initial onset of neurological symptoms in multiple sclerosis, JAMA 293 (2005) (20), pp. 2496-2500.

[2] G.C. Ebers, A.D. Sadovnick and N.J. Risch, A genetic basis for familial aggregation in multiple sclerosis, Nature 377 (1995), pp. 150-151.

[3] A. Feix, W.C. Winkelmayer, C. Eberle, G. Sunder-Plassmann and M. Fodinger, Methionine synthase reductase MTRR 66A > G has no effect on total homocysteine, folate, and Vitamin B12 concentrations in renal transplant patients, Atherosclerosis 174 (2004), pp. 43-48.

[4] D.J. Gaughan, L.A.J. Kluijtmans, S. Barbaux, D. McMaster, I.S. Young and J.W.G. Yarnell et al., The methionine synthase reductase (MTRR) A66G polymorphism is a novel genetic determinant of plasma homocysteine concentrations, Atherosclerosis 157 (2001), pp. 451-456.

[5] D. Leclerc, A. Wilson, R. Dumas, C. Gafuik, D. Song and D. Watkins et al., Cloning and mapping of a cDNA for methionine synthase reductase, a flavoprotein defective in patients with homocystinuria, Proc Natl Acad Sci U S A 95 (1998), pp. 3059-3064.

[6] A.M. Troen, The central nervous system in animal models of hyperhomocysteinemia, Prog NeuroPsychopharmacol Biol Psychiatry 29 (2005) (7), pp. 1140-1151.

[7] R. Rozen, Genetic predisposition to hyperhomocysteinemia: deficiency of methylenetetrahydrofolate reductase (MTHFR), Thromb Haemost 78 (1997) (1), pp. 523-526.

[8] N.M. van der Put, F. Gabreels, E.M. Stevens, J.A. Smeitink, J.F. Trijbels and K.T. Eskes et al., A second common mutation in the methylenetetrahydrofolate reductase gene: an additional risk factor for neural-tube defects, Am J Hum Genet 62 (1998), pp. 1044-1051.

[9] H. Olteanu, T. Munson and R. Banerjee, Differences in the efficiency of reductive activation of methionine synthase and exogenous electron acceptors between the common polymorphic variants of human methionine synthase reductase, Biochemistry 41 (2002), pp. 13378-13385.

[10] I. Kara, A. Sazci, E. Ergul, G. Kaya and G. Kilic, Association of the C677T and A1298C polymorphisms in the 5,10-methylenetetrahydrofolate reductase gene in patients with migraine risk, Mol Brain Res 111 (2003), pp. 84-90.

[11] B.M. Keegan and J.H. Noseworthy, Multiple Sclerosis, Annu Rev Med 53 (2002), pp. 285-302.

[12] S.A. Lipton, W.K. Kim, Y.B. Choi, S. Kumar, D.M. D'Emilia and P.V. Rayudu et al., Neurotoxicity associated with dual actions of homocysteine at the $N$-methyl-d-aspartate receptor, Proc Natl Acad Sci 94 (1997), pp. 5923-5928.

[13] Z. Chen, B. Ge, T.J. Hudson and R. Rozen, Microarray analysis of brain RNA in mice with methylenetetrahydrofolate reductase deficiency and hyperhomocysteinemia, Gene Expr Patterns 1 (2002), pp. 89-93.

[14] C. Kruman, C. Culmsee, S.L. Chan, Y. Kruman, Z. Guo and L. Penix et al., Homocysteine elicits a DNA damage response in neurons that promotes apoptosis and hypersensitivity to excitotoxicity, $J$ Neurosci 20 (2000), pp. 6920-6926.

[15] M. Fodinger, H. Buchmayer and G. Sunder-Plassmann, Molecular genetics of homocysteine metabolism, Miner Electrolyte Metab 25 (1999), pp. 269-278.

[16] J.W. Muntjewerff, R.S. Kahn, H.J. Blom and M. den Heijer, Homocysteine, methylenetetrahydrofolate reductase and risk of schizophrenia: a meta-analysis, Mol Psychiatry 11 (2006) (2), pp. 143-149.

[17] E. Susser, A.S. Brown, E. Klonowski, R.H. Allen and J. Lindenbaum, Schizophrenia and impaired homocysteine metabolism: a possible association, Biol Psychiatry 44 (1998) (2), pp. 141-143.

[18] J. Lindenbaum, E.B. Healton, D.G. Savage, J.C. Brust, T.J. Garrett and E.R. Podell et al., Neuropsychiatric disorders caused by cobalamin deficiency in the absence of anemia or macrocytosis, NEJM 318 (1988) (26), pp. 1720-1728.

[19] G.S. Ramsaransing, M.R. Fokkema, A. Teelken, A.V. Arutjunyan, M. Koch and J. De Keyser, Plasma homocysteine levels in multiple sclerosis, J Neurol Neurosurg Psychiatry 77 (2006) (2), pp. 189-192.

[20] S.M. Baig and G. Ali Qureshi, Homocysteine and vitamin B12 in multiple sclerosis, Biog Amines 11 (1995), pp. 479-485.

[21] M. Vrethem, E. Mattsson, H. Hebelka, K. Leerbeck, A. Osterberg and A.M. Landtblom et al., Increased plasma homocysteine levels without signs of vitamin B12 deficiency in patients with 
multiple sclerosis assessed by blood and cerebrospinal fluid homocysteine and methylmalonic acid, Mult Scler 9 (2003) (3), pp. 239-245.

[22] H.T. Besler and S. Comoglu, Lipoprotein oxidation, plasma total antioxidant capacity and homocysteine level in patients with multiple sclerosis, Nutr Neurosci 6 (2003) (3), pp. 189-196.

[23] P.I. Ho, D. Ortiz, E. Rogers and T.B. Shea, Multiple aspects of homocysteine neurotoxicity: glutamate excitotoxicity, kinase hyperactivation and DNA damage, J Neurosci Res 70 (2002) (5), pp. 694-702. 